\title{
Infiltración testicular por leucemia linfoblástica aguda, presentándose como masa testicular: reporte de un caso
}

Sandra Herrera-Lomonaco ${ }^{1, a}$, Marticela Cabeza-Morales ${ }^{2, a}$, Indira Angulo-Díaz ${ }^{2, a}$, Zenen Carmona-Meza ${ }^{3, a}$, Hugo Rafael Corrales-Santander ${ }^{4}$

\section{RESUMEN}

La leucemia linfoblástica aguda es una neoplasia predominante en pediatría, corresponde al $11 \%$ de las leucemias. En Colombia hay una incidencia anual en adultos de 1 por cada 100.000 habitantes. La recaída testicular es rara alcanzando solo del 0.9 a $8.8 \%$ de todos los casos y representa un signo de recurrencia que puede ser confundida con otros padecimientos testiculares. Se reporta el caso de un paciente masculino de 23 años de edad, con cuadro de 2 meses de evolución consistente en dolor y aumento de tamaño testicular, posterior a trauma, con antecedente de leucemia linfoblástica aguda tratada hace 4 años. Se realiza ecografía testicular que sugiere hidrocele a tensión. Es llevado a cirugía, donde se aprecia testículo con características neoplásicas por lo cual se realiza orquiectomía. Resultado de anatomía patológica concluye masa $12 \mathrm{~cm}$ correspondiente a infiltración por leucemia aguda, CD10 y CD34 positivo y Tdt negativo.

El testículo es considerado santuario de células leucémicas, sin embargo excepcionalmente puede observarse formación de una masa simulando un tumor testicular.

Palabras clave: Leucemia-linfoma linfoblástico; neoplasias testiculares; leucemia.

\section{Testicular infiltration due to acute lymphoblastic leukemia presented as a testicular mass: a case report}

\section{ABSTRACT}

Acute Iymphoblastic leukemia is a common pediatric neoplasia, comprising $11 \%$ of all leukemias. In Colombia, the annual incidence in adults is 1 out of 100,000 people. Testicular relapse is rare (accounting for only 0.9-8.8\% of all cases) and represents a sign of recurrence which can be confused with other testicular conditions. We report the case of a 23-yearold male with testicular pain and swelling for 2 months after a trauma, and a history of acute lymphoblastic leukemia treated 4 years before. A testicular ultrasound suggested tension hydrocele. The patient was taken to surgery where a testis with neoplastic features was observed. Therefore, an orchiectomy was performed. The pathological anatomy revealed a 12-cm mass consisting of infiltration by leukemic cells, showing a positive expression for CD19, CD10, and a negative expression for TdT.

The testes are considered a sanctuary site for leukemic cells. However, the formation of a mass that mimic a testicular tumor may exceptionally be observed.

Keywords: Lymphoblastic leukemia/lymphoma; testicular neoplasia; leukemia.

1. Médico Patóloga.

2. Estudiante de medicina.

3. J efe Departamento de Investigaciones.

4. Médico, Coordinador grupo de investigación Centro de Investigaciones Biomédicas.

a. Facultad de Medicina, Universidad de Cartagena. 


\section{INTRODUCCIÓN}

La leucemia linfoblástica aguda (LLA) es el tipo de cáncer más frecuente en la niñez ${ }^{(1)}$, es rara su presentación fuera de este rango etáreo ${ }^{(2)}$. Es definida por la presencia de más de $20 \%$ de linfoblastos en la médula ósea o sangre periférica ${ }^{(3)}$. Puede presentar recaídas luego de su efectivo tratamiento. El testículo es el segundo sitio de recaída extramedular más común; pese a esto la enfermedad testicular clínicamente demostrable al momento del diagnóstico, es poco frecuente ${ }^{(2,4)}$. Además suele presentarse en etapas avanzadas de la enfermedad ${ }^{(5)}$.

El obj etivo de este estudio es presentar un caso de recidiva de la leucemia linfoblástica aguda, que se manifestó como una masa testicular, y que posterior a su intervención, presentó deterioro abrupto de su condición clínica.

\section{CASO CLÍNICO}

Paciente masculino de 23 años de edad que acude al servicio de urgencias por un cuadro clínico de 2 meses de evolución consistente en dolor en flanco derecho irradiado a testículo ipsilateral, tratado como un cólico renal, con antiespasmódicos, con mejoría parcial. Un mes después consulta nuevamente por reaparición de la sintomatología, adicionalmente refiere un aumento del tamaño del testículo afectado. Como antecedente de importancia manifiesta leucemia linfoblástica aguda, tratada hace 5 años con resolución aparente, además refiere un trauma testicular moderado, que coincide con el inicio del cuadro clínico. Al examen físico se encuentran signos de inflamación, aumento del contenido escrotal derecho, orquialgia y dificultad para pal pación de testículo con transiluminación negativa, testículo izquierdo sin alteraciones. Ante estos hallazgos y en vista de la localización y características de la masa, se plantean los siguientes diagnóstico diferenciales: hidrocele a tensión, hernia inguinal, tumor germinal y linfoma ${ }^{(2)}$.

Para la confirmación de la sospecha clínica se decide realizar un cuadro hemático (Tabla 1), y Eco Doppler, el cual sugiere un hidrocele a tensión, por ende se decide realizar manejo quirúrgico mediante hidrocelectomía.

Tabla 1. Cuadro hemático realizado previo a la intervención quirúrgica

\begin{tabular}{|cc|}
\hline Parámetros & Valores \\
\hline Hemoglobina & $13 \mathrm{Gr}$ \\
\hline Hematocrito & $38 \%$ \\
\hline Leucocitos & $20890 \mathrm{~mm}^{3}$ \\
\hline Plaquetas & $17100 \mathrm{~mm}^{3}$ \\
\hline
\end{tabular}

Durante la intervención se observa hidrocele, con testículo derecho que presenta cambios inflamatorios crónicos, con consistencia indurada y abundantes áreas de necrosis y equimosis, considerándolo no viable, y sospechoso de malignidad, motivo por el cual se decide realizar orquiectomía simple derecha, previo consentimiento informado (Figura 1 y 2 ).

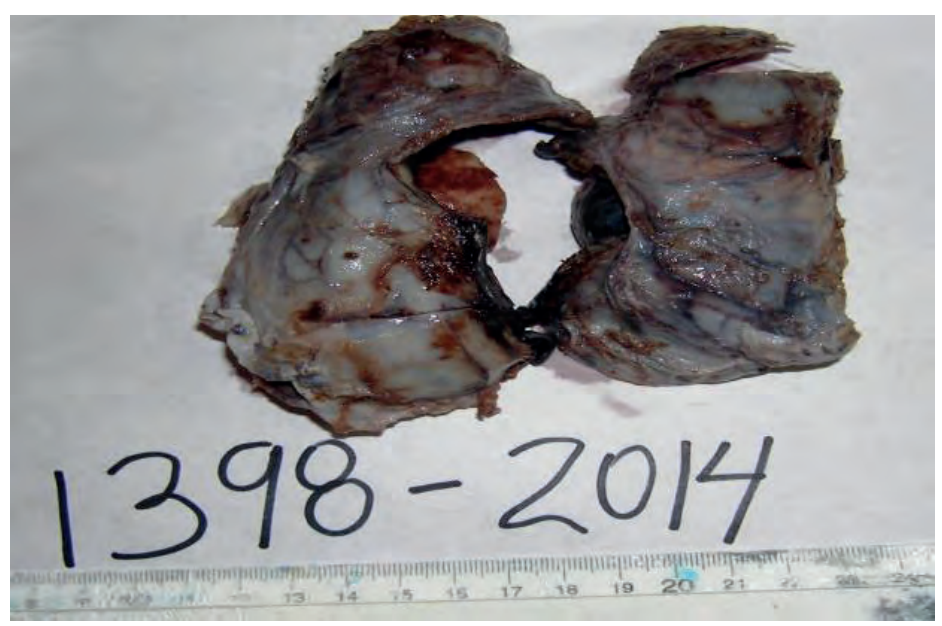

Figura 1. Testículo derecho con superficie externa corresponde a túnica vaginalis despulida, con áreas de necrosis y hemorragias 


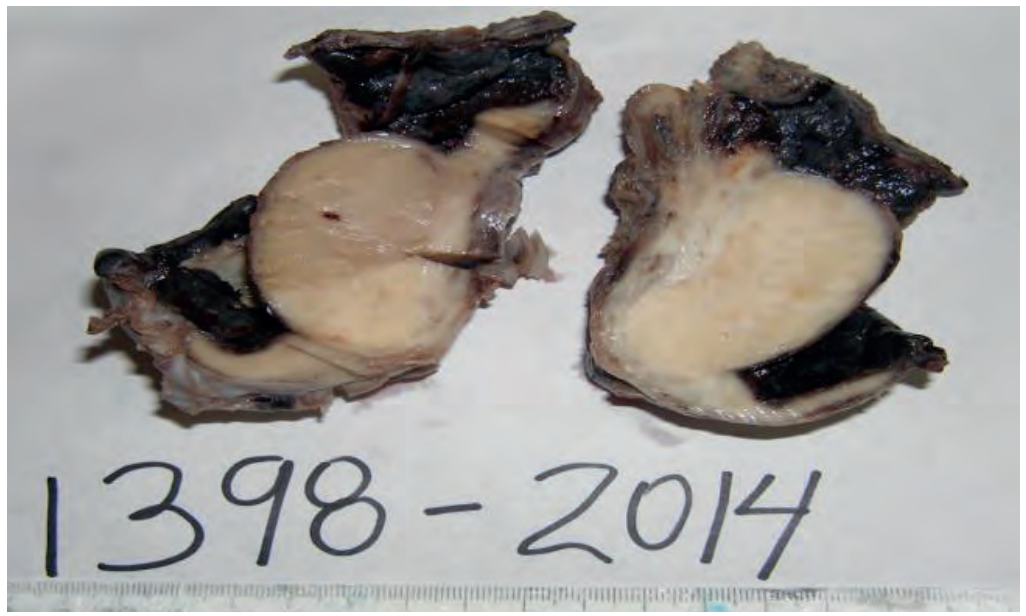

Figura 2. Testículo que mide $12 \times 7 \times 5 \mathrm{~cm}$, al corte se identifica una masa carnosa, amarillenta, bien delimitada que mide $6 \times 5 \times 5$ $\mathrm{cm}$, solida rodeada de un área cavitada con escaso material hemorrágica. Consistencia firme, sólida, carnosa, no es posible identificar elementos del cordón

El paciente toleró bien el procedimiento y es dado de alta con antibióticos. Reporte de Anatomía Patológica muestra neoplasia linfoide, constituida por linfocitos monótonos, de apariencia clonal, que comprometen el intersticio testicular, túnica vaginalis, epidídimo y elementos del cordón de manera difusa y extensa. Se observan túbulos seminíferos parcialmente obliterados, con células germinales en su luz, rodeados por linfocitos neoplásicos, y extensas áreas de necrosis. Los márgenes de sección están comprometidos por la lesión. No se reconoce parénquima testicular sano, ni elementos epiteliales, granulomas ni abscesos (Figura 3 y 4 ).

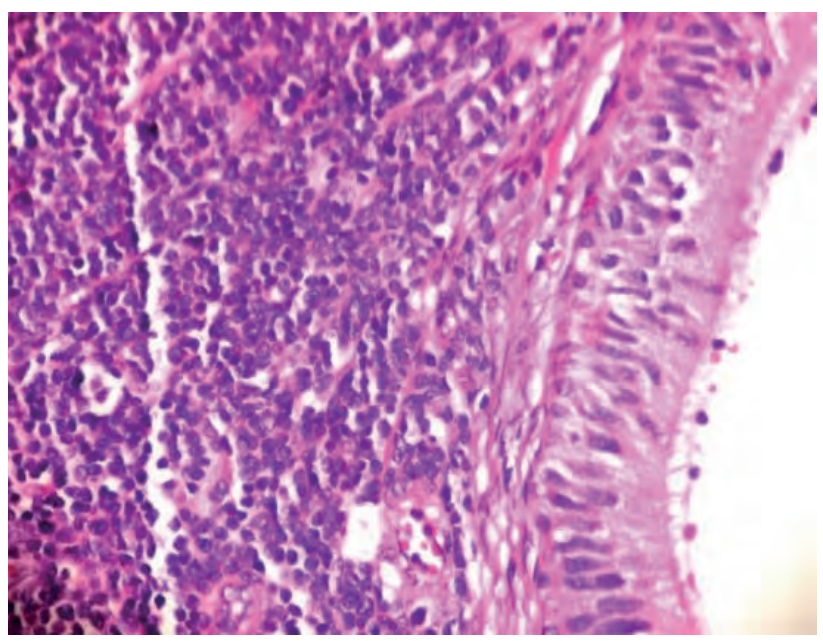

Figura 3. Imagen microscópica que muestra una proliferación de células pequeñas de aspecto linfoide, monótonas, de apariencia clonal. Infiltrando parénquima testicular. En el lado derecho se observa compromiso del epidídimo

Se identifica presencia de hidrocele asociado. Ante estos hallazgos se plantea el diagnóstico diferencial entre un linfoma no Hodgkin tipo linfoma linfoblástico y una infiltración leucémica secundaria. Los marcadores de Inmunohistoquímica reportaron positividad para CD10 y CD34 y Tdt negativo, confirmando el diagnóstico de Leucemia Linfoblástica aguda fenotipo B. El paciente asiste a consulta control posoperatoria a los 15 días donde se encuentra dolor, hematoma, dehiscencia de sutura y signos de infección local del sitio operatorio, por lo cual se hospitaliza. Se realiza valoración por medicina interna y oncología clínica, para continuar manej o. Paraclínicos muestra leucocitos 127.760/ $\mathrm{mm} 3$, con $99 \%$ de células inmaduras, anemia (Hemoglobina de $5,8 \mathrm{~g}$ ) y trombocitopenia (33000/ mm3), BUN $24 \mathrm{mg} / \mathrm{dL}$, Creatinina $2.7 \mathrm{mg} / \mathrm{dL}$, TGO $21 \mathrm{U} / \mathrm{L}$, TGP $21 \mathrm{U} / \mathrm{L}, \mathrm{LDH}: 819$ UI/L. Se traslada a la unidad de aislados para pacientes inmunocomprometidos, con antibioticoterapia intravenosa y cuidados generales. 


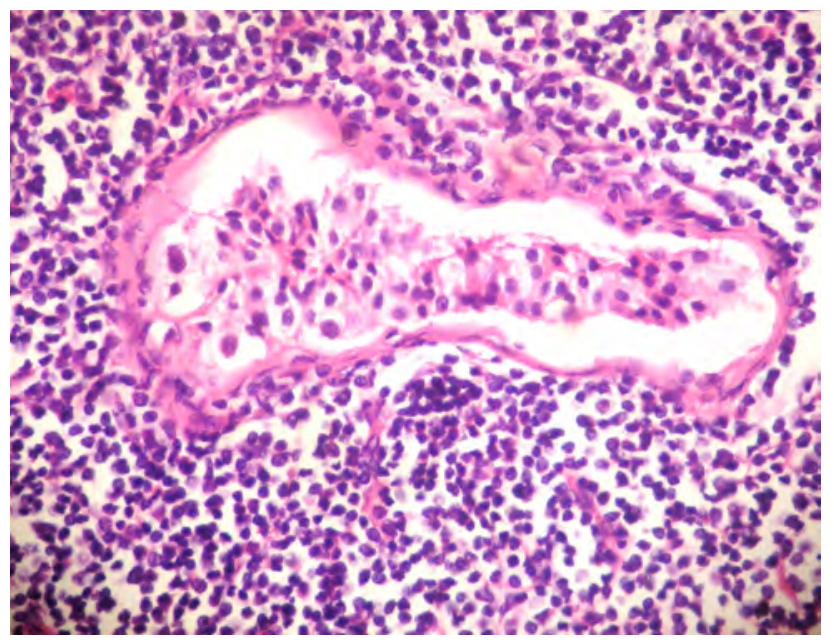

Figura 4. Imagen microscópica que muestra infiltración leucémica, difusa del intersticio testicular. Se observa un túbulo seminífero obliterado rodeado por células tumorales

El paciente presenta una tórpida evolución, con presencia de taquicardia, edema generalizado con signos de falla renal aguda desarrollando síndrome de lisis tumoral y deterioro clínico. Es remitido a una unidad oncológica de quinto nivel para manejo interdisciplinario, donde recibe esquema de quimioterapia para Leucemia linfoblástica aguda, con mejoría sustancial de su cuadro. Actualmente se encuentra estable, y continua en protocolo de seguimiento.

\section{DISCUSIÓN}

La leucemia linfoblástica aguda presenta una incidencia anual de 2.5 a 3 casos por cada 10.000 niños menores de 15 años, con pico en la edad escolar y predominio masculino. Nuestro paciente escapa un poco de esta frecuencia, debido a que se le presentó a los 18 años de edad. La recurrencia testicular tiene una frecuencia baja de 0.9 a $8.8 \%{ }^{(2)}$.

Etiológicamente la LLA está dada por una mutación somática de la célula progenitora linfoide, lo que altera su división normal, generando un crecimiento anormal de células inmaduras de los componentes sanguíneos (blastos), cuya acumulación se acompaña de una disminución del tejido hematopoyético normal en médula ósea y posterior invasión de sangre periférica y otros tejidos ${ }^{(3)}$. Se han descrito algunos lugares llamados "santuarios", que son sitios anatómicos que sirven como lugar de almacenamiento de las células leucémicas, donde se encuentran protegidas de los agentes quimioterapéuticos; los más comunes son testículo y Sistema nervioso central, debido a la presencia de las barreras hematoencefálica y hematotesticular, respectivamente, las cuales aíslan estos tejidos de elementos de la sangre que podrían alterar la función de los mismos ${ }^{(6,7)}$. Los infiltrados leucémicos se han detectado de manera frecuente en autopsias, en el $40-65 \% \%$ de los pacientes con leucemia aguda y hasta un $35 \%$ en leucemia crónica ${ }^{(2)}$. El mecanismo mediante el cual el tumor migra, no se ha descrito completamente ${ }^{(3)}$, se cree que puede ser por su almacenamiento en lugares "santuario". Sin embargo, estudios recientes utilizando reacciones de cadena de polimerasa (PCR) para identificar secuencias específicas de leucemia han evidenciado que las células malignas pueden estar presentes en la médula ósea al momento de la recurrencia extramedular, aunque a la microscopia se observe remisión medular de la enfermedad ${ }^{(2)}$. En las recaídas el infiltrado leucémico no suele ser de gran tamaño, por lo cual el diagnóstico en biopsia es complicado. Generalmente cursa con un patrón de infiltración intersticial, similar al linfoma, por lo cual se hace necesaria la evaluación de sangre periférica y médula ósea para establecer el diagnóstico diferencial ${ }^{(2,4)}$.

El cuadro clínico de la LLA, abarca signos y síntomas agudos o insidiosos, que incluyen: fiebre, fatiga, dolor óseo, artralgia, dolor de cabeza, vómitos y alteración del estado mental (3). Los cuales son una manifestación clínica de eventos que se desarrollan en el curso de la enfermedad como son anemia, neutropenia y trombocitopenia subyacentes secundarias a la invasión de la médula ósea y de otros tejidos. Es muy interesante ver que en nuestro paciente al momento de detectarse la masa testicular no presentaba alteraciones hematológicas de consideración, y si bien se notó un incremento en el número de glóbulos blancos, este hallazgo fue considerado reactivo en ausencia de blastos en sangre periférica (Tabla 1) ${ }^{(3)}$, Io cual hace más interesante su debut como masa testicular. Solo después de la intervención quirúrgica se presentaron las manifestaciones hematológicas y sistémicas floridas, por lo que consideramos el procedimiento quirúrgico como 
factor estresante que exacerbó el cuadro e hizo florida la sintomatología. Es importante mencionar que el paciente presentó un cuadro compatible con síndrome de lisis tumoral. Este síndrome es una emergencia metabólica que generalmente es producto de una masiva destrucción de células neoplásicas y se caracteriza por hiperuricemia, hiperfosfatemia, hiperkalemia, hipocalcemia, acidosis láctica y azoemia ${ }^{\left({ }^{8}\right)}$. Esta entidad es vista comúnmente como una complicación del tratamiento de neoplasias hematológicas, sensibilidad aumentada a la quimioterapia y altas tasas de proliferación.

Un alto conteo de células blancas, niveles séricos de LDH aumentados, enfermedad renal previa e hiperuricemia, son factores de riesgo para el desarrollo de síndrome tumoral. Sin embargo, el síndrome de lisis tumoral puede desarrollarse de forma espontánea, aunque con muy poca frecuencia ${ }^{(9)}$. El tratamiento incluye hidratación intravenosa agresiva con solución salina y diuréticos de asa para mantener un adecuado gasto urinario. El alopurinol se utiliza para disminuir la concentración de ácido úrico en plasma. En casos en los que el paciente presenta una hiperkalemia que amenaza la vida del mismo o una sobrecarga de volumen y tienen una oliguria persistente a pesar de la hidratación agresiva, estos deben ser manejados con hemodiálisis ${ }^{(8,9)}$. El paciente de este caso presentaba factores de riesgo para desarrollar un síndrome de lisis tumoral, como el conteo alto de leucocitos, cifra incrementada de BUN y niveles aumentados de LDH. Además, este paciente fue sometido a una intervención quirúrgica que pudo jugar un papel de factor estresante que pudiera desencadenar la complicación ya mencionada, pero la causa exacta de dicha complicación es aún incierta.

Ante un incremento en el volumen testicular existe un amplio rango de posibilidades diagnósticas, en lo cual es importante tener en cuenta la edad y los antecedentes del paciente. Las causas más comunes de incremento del volumen testicular son: hidrocele, que cursa con transiluminación positiva; hernias inguinales, las cuales se acompañan de dolor que se desencadena con el ejercicio, y gran incomodidad; varicocele, que se caracteriza por la presencia de una vena dilatada observable o detectable por palpación; además de tumores de tipo germinal y no germinal ${ }^{(2)}$. En caso de que exista dolor, rubor y calor, se deben sospechar patologías como: orquitis, epididimitis y celulitis escrotal ${ }^{(2)}$.

El diagnóstico de la LLA se realiza mediante estudios en sangre periférica 0 médula ósea ${ }^{(3)}$, acompañado de estudios moleculares. En su presentación como masa testicular se recomienda realizar biopsia testicular en la cual se puede observar infiltración en el tejido testicular con predominio de infiltración intersticial y no en los túbulos ${ }^{(4)}$. Se ha demostrado que la detección por reacción en cadena de polimerasa (PCR) es más sensible ${ }^{(2)}$. Estas medidas no fueron aplicables a nuestro caso, debido a que fue descubierta incidentalmente cuando era intervenida como hidrocele a tensión con dolor.

El abordaje de estos pacientes con cuadros de recidiva implica hacer un tratamiento sistémico con quimioterapia, debido a que si se trata en forma local con radioterapia, es muy probable el desarrollo de recurrencia medular (2). En nuestro caso el paciente ameritó tratamiento quirúrgico inicial por la sintomatología clínica dolorosa y la presentación imagenológica como un hidrocele a tensión, lo cual implicó mayor número de medidas, por el consecuente desarrollo de síndrome de lisis tumoral, junto con otras complicaciones ya expuestas. Se debe tener en cuenta que en este tipo de terapias (quimioterapia y extracción quirúrgica), en pacientes jóvenes los exponen a perdida de la espermatogénesis ${ }^{(10,11)}$, alterando su función reproductiva, especialmente si se usan fármacos como metrotexate (2), cistaplatino, ciclofosfamida, procarbazina ${ }^{(12)}$; lo ideal en estos pacientes es realizar crio-preservación, para que posteriormente pueda conservar su función reproductiva ${ }^{(12-14)}$. Además vale la pena mencionar que la viabilidad, motilidad, y morfología de estos espermatozoides es muy buena cuando el tumor es de tipo hematológico, diferente a lo que ocurre cuando su origen es germinal ${ }^{(13,14)}$. Es importante resaltar que en este caso, no se consideró este aspecto ya que el testículo estaba notablemente afectado, con extensa necrosis que lo hacía inviable.

La prevención ha sido sugerida, mediante la implementación de biopsia testicular bilateral durante el tratamiento de mantenimiento o inmediatamente antes del cese electivo de la quimioterapia. Ésta práctica ha sido cuestionada, debido a que algunos estudios indican que las biopsias testiculares al momento del diagnóstico, después de la inducción, durante el mantenimiento 0 antes del cese electivo del tratamiento, están asociadas con resultados falsos negativos y no son predictores seguros para determinar una eventual recaída a nivel testicular ${ }^{(4)}$

El pronóstico de estos pacientes es bueno, suelen cursar con una sobrevida libre de enfermedad en el $70 \%$ de los casos $^{(2)}$. Este porcentaje varía según la edad, respuesta al tratamiento, anomalías citogenéticas y velocidad de respuesta a la terapia ${ }^{(3)}$.

\section{REFERENCIAS BIBLIOGRÁFICAS}

1. Borgmann A, Zinn C, Hartmann R, Herold R, Kaatsch P, Escherich G, et al. Secondary malignant neoplasms after intensive treatment of relapsed acute lymphoblastic leukaemia in childhood. Eur J Cancer. 2008;44(2):257-268.

2. Río R Del, Willatt SJ, Villanueva J, Vega O, Truan D. Aumento de volumen testicular en pacientes con leucemia linfoblástica aguda. Rev Ped Elec. 2006;3(1):8-11. 
3. ACS: American Cancer Society [Internet]. Atlanta: ACS [actualizado 03/03/2016; citado 29/11/2016]. Disponible en: http:// www. cancer.org/ acs/ groups/ cid/ documents/ webcontent/ 002302-pdf. pdf.

4. Dorbeker-Azcona R, Cárdenas-Cardós R, Braun-Roth G, MoralesHernández E. Diagnóstico de infiltración testicular por medio de biopsia por aspiración con aguja fina en pacientes con leucemia aguda. GAMO. 2009;8(5):69-74.

5. Ibarguren RL, Sastre VA, Padilla J, Pertusa C. Tumores no germinales de testículo. Arch Esp Urol. 2005;58(10):1031-1034.

6. Engelhardt S, Patkar S, Ogunshola 00. Cell-specific blood-brain barrier regulation in health and disease: a focus on hypoxia. $\mathrm{Br}$ J Pharmacol. 2014;171(5):1210-1230.

7. Pereira ML, Garcia e Costa F. The blood-testis barrier as a target of some chemotherapeutic agents. Chemotherapy. 2007;53(6): 446-448.

8. Akoz AG, Yildirim N, Engin H, Dagdas S, Ozet G, Ozel T, et al. An unusual case of spontaneous acute tumor lysis syndrome associated with acute lymphoblastic leukemia: a case report and review of the literature. Acta Oncol. 2007;46(8):11901192.

9. Latha SM, Krishnaprasadh D, Murugapriya P, Scott JX. Single dose rasburicase in the management of tumor lysis syndrome in childhood acute lymphoblastic leukemia: A case series. Indian J Nephrol. 2015; 25(2): 91-94.

10. Magelssen $H$, Haugen TB, von Düring V, Melve KK, Sandstad B, Fosså SD. Twenty years experience with semen cryopreservation in testicular cancer patients: who needs it? Eur Urol. 2005; 48(5): 779-785.

11. Lambert SM, Fisch $\mathrm{H}$. Infertility and testis cancer. Urol Clin North Am. 2007;34(2):269-277.

12. Trottmann M, Becker AJ , Stadler T, Straub J, Soljanik I, Schlenker $B$, et al. Semen quality in men with malignant diseases before and after therapy and the role of cryopreservation. Eur Urol. 2007; 52(2):355-367.
13. Degl'Innocenti S, Filimberti E, Magini A, Krausz C, Lombardi $G$, Fino $M$, et al. Semen cryopreservation for men banking for oligospermia, cancers, and other pathologies: prediction of post-thaw outcome using basal semen quality. Fertil Steril. 2013; 100(6): 1555-1563.

14. Williams DH, Karpman E, Sander JC, Spiess PE, Pisters LL, Lipshultz LI. Pretreatment semen parameters in men with cancer. J Urol. 2009; 181(2):736-740.

\section{Fuentes de financiamiento:}

Este artículo ha sido financiado por los autores.

\section{Conflictos de interés:}

Los autores declaran no tener ningún conflicto de interés.

\section{Correspondencia:}

Sandra Herrera Lomonaco

Dirección: Urbanización la Española, Manzana A, Lote 5

Teléfono: 3126230951 - 6454949

Correo electrónico: sandraherreralomonaco@gmail.com

Recibido: 09 de noviembre de 2016 Aprobado: 29 de noviembre de 2016 